\title{
Correspondence
}

\section{A Novel Image Recovery Algorithm for Visible Watermarked Images}

\author{
Soo-Chang Pei and Yi-Chong Zeng
}

\begin{abstract}
A novel image recovery algorithm for removing visible watermarks is presented. Independent component analysis (ICA) is utilized to separate source images from watermarked and reference images. Three independent component analysis approaches are examined in the proposed algorithm, which includes joint approximate diagonalization of eigenmatrices, second-order blind identification, and FastICA. Moreover, five different visible watermarking methods to embed uniform and linear-gradient watermarks are implemented. The experimental results show that visible watermarks are successfully removed, and that the proposed algorithm is independent of both the adopted ICA approach and the visible watermarking method. In the final experiment, several public domain images sourced from various websites are tested. The results of this study demonstrate that the proposed algorithm can blindly and successfully remove the visible watermarks without knowing the watermarking methods in advance.
\end{abstract}

Index Terms-Image recovery, independent component analysis (ICA), visible watermark removal.

\section{INTRODUCTION}

Various image recovery schemes have been developed recently and used in digital photograph restoration [1], [2], ancient painting restoration [3], and visible watermark removal [4]. Image recovery attempts to fill the selected area with the appropriate textures, where the undesired object was initially located. There are two kinds of the undesired object, which are: 1) the undesired solid object occluding the background and 2) the undesired transparent object merging with the background.

Reviewing previous studies on solid objects removal, Bertalmio et al. developed the image-inpainting approach [1] for filling a selected area with the surrounding pixels. Sun et al. suggested a structure propagation method for image completion, and adopted patch-based texture synthesis to restore selected areas [2]. A texture synthesis scheme is also utilized in the virtual restoration of ancient Chinese paintings [3] by adding some auxiliaries to improve the method. In the transparent object removal, Huang and $\mathrm{Wu}$ employed the image-inpainting approach to remove visible watermarks [4]. However, the iterative process of image-inpainting is costly and time-consuming.

This work presents a fast, simple, and efficient image recovery algorithm for removing visible watermarks. The rest of this paper is organized as follows. Section II briefly introduces the related methods. Section III then describes the visible watermark removal algorithm.

Manuscript received November 11, 2005; revised May 3, 2006. This work was supported by the National Science Council of Taiwan, R.O.C., under Contract NSC94-2213-E-002-072 and Contract NSC93-2752-E-002-006-PAE. The assoicate editor coordinating the review of this manuscript and approving it for publication was Prof. Mohan S. Kankanhalli.

S.-C. Pei is with the Department of Electrical Engineering, National Taiwan University, Taipei 10617, Taiwan, R.O.C. (e-mail: pei@cc.ee.ntu.edu.tw).

Y.-C. Zeng is with the Graduate Institute of Communication Engineering, National Taiwan University, Taipei 10617, Taiwan, R.O.C. (e-mail: d89942010@ntu.edu.tw).

Color versions of Figs. 1 and 6-8 are available online at http://ieeexplore. ieee.org.

Digital Object Identifier 10.1109/TIFS.2006.885031
Section IV presents the experimental results. Conclusions are drawn in Section V.

\section{RELATED Methods}

\section{A. Visible Watermarking Methods}

A visible watermark represents the owner of the product. Many online images, digital documents, and video are embedded with visible watermarks. Braudaway et al. embedded visible watermarks to protect public images [5]. They formulate the nonlinear equation to accomplish the luminance alteration on the pixel domain, and then the watermark is placed onto the image. In addition, various parameters are adopted in the nonlinear equation in order to make the watermark difficult to remove. Meng and Chang added visible watermarks to video sequence in the discrete cosine transform (DCT) domain [6]. To extend the Braudaway's method, they developed the simple stochastic approximation model on the DCT domain and modified the original nonlinear equation. The feature is to directly implement the adaptive watermarking technique to the compressed video steams. Mohanty et al. proposed a watermarking method in the DCT domain [7] by combining a visible and invisible watermarks as a dual watermark to be embedded into an image [8]. $\mathrm{Hu}$ and Kwong implemented an adaptive visible watermarking in the wavelet domain [9]. Both the host image and watermark are first decomposed to a four-level multiresolution wavelet-based structure. In each subband, the scaling factors of the wavelet coefficients are calculated, and then the coefficients of the watermark are imposed on those of the host image with the scaling factors. Chen developed a visible watermarking mechanism in the pixel domain based on the statistical approach [10]. The mechanism is different from Braudaway's method in the watermarked ratio, which is determined by the standard deviation of the block. Regardless of exploiting the visible watermarking technique, the watermarked image can generally be formulated as

$$
y_{w}(i, j)=k_{1}(i, j) x(i, j)+k_{2}(i, j) w(i, j)
$$

where $x(i, j), y_{w}(i, j)$ and $\mathrm{w}(i, j)$ are the $(i, j)$ th pixels of the host image, the watermarked image, and the visible watermark, respectively, and $k_{1}(i, j)$ and $k_{2}(i, j)$ are the two weighting factors. In most of the visible watermarking methods, all weighting factors are usually variable.

\section{B. Independent Component Analysis (ICA)}

ICA is also called blind signal separation (BSS). It is a statistical and computational scheme that yields the latent correlation of a set of random variables or signals. The simplest independent component analysis (ICA) model assumes that $m$ sources $\left(s_{1}, s_{2}, \ldots, s_{m}\right)$ generate $m$ mixtures $\left(t_{1}, t_{2}, \ldots, t_{m}\right)$

$$
t_{k}=a_{k, 1} s_{1}+a_{k, 2} s_{2}+\cdots+a_{k, m} s_{m}
$$

where $a_{k, l}$ denotes the mixing value of the $l$ th independent source in the $k$ th mixture, and $1 \leq k, l \leq m$. The ICA estimates the separating value $b_{k, l}$, such that the approximate sources $\hat{s}_{k}$ can be separated from the mixtures, and $\hat{s}_{k}=b_{k, 1} t_{1}+b_{k, 2} t_{2}+\cdots+b_{k, m} t_{m}$.

Cardoso and Souloumiac presented a blind identification algorithm by the joint approximate diagonalizaition of eigenmatrices (JADE) to estimate the separating matrix [11]. FastICA, proposed by Hyvarinen 


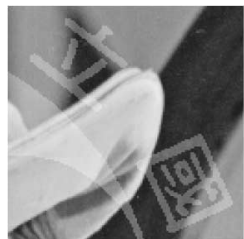

(a)

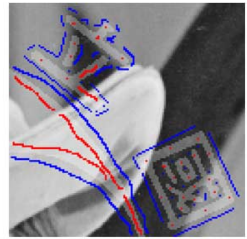

(b)

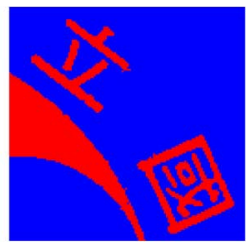

(c)
Fig. 1. Segment the watermark area using the seed-growing algorithm. (a) The watermarked image. (b) The marked points for seed growing are added on the watermarked image as indicated. (c) The segmented-watermarked area.

and Oja [12], is an ICA approach with fast convergence. Belouchrani et al. exploited stationary second-order statistics based on a joint diagonalization of a set of covariance matrices to solve the ICA problem, which is called second-order blind identification (SOBI) [13].

To analyze (1), the host image to be separated from the watermarked image corresponds to solve the ICA problem of two independent sources in (2). However, only a mixture (the watermarked image $\mathrm{y}_{\mathrm{w}}$ ) is available, another mixed image (the reference image $y_{f}$ ) must be generated for the ICA. Section III further discusses those details.

\section{Visible WATERMARK REMOVAL AlgORITHM (VWR)}

The proposed VWR has three phases: watermarked area segmentation, reference image generation, and image recovery. These phases are described below.

\section{A. Watermarked Area Segmentation}

Before the image recovery algorithm is implemented, the visible watermark must be segmented. First, the user sets red and blue marked points manually to represent the watermarked and the nonwatermarked pixels, respectively. For instance, a watermarked image is shown in Fig. 1(a) and the marked points for seed growing are added on the watermarked image as shown in Fig. 1(b). Subsequently, the seed-growing algorithm is exploited to turn all pixels into the marked points. Let $p(i, j)$ be the $(i, j)$ th marked point and $p_{\mathrm{N}}(i, j, n)$ be the $n$th neighbor unmarked point of $p(i, j)$. The intensity difference between $p(i, j)$ and $p_{\mathrm{N}}(i, j, n)$ is defined as

$$
\operatorname{diff}\left(\mathbf{I}(p(i, j)), \mathbf{I}\left(p_{\mathrm{N}}(i, j, n)\right)\right)=\left|\mathbf{I}(p(i, j))-\mathbf{I}\left(p_{\mathrm{N}}(i, j, n)\right)\right|
$$

where $\mathbf{I}(p)$ denotes the intensity of $p$. If the intensity distance is smaller than the threshold $\tau$, then $p_{\mathrm{N}}(i, j, n)$ will be designated as a red marked point when $p(i, j)$ is a red marked point, or $p_{N}(i, j, n)$ will be designated as the blue marked point since $p(i, j)$ is a blue marked point. However, the seed-growing algorithm may be stagnated; the solution is to increase the threshold $\tau$ with the increment $\Delta \tau$ (i.e., $\tau \leftarrow \tau+\Delta \tau$ ). Fig. 1(c) represents the segmented-watermarked area.

\section{B. Reference Image Generation}

Based on the description in Section II-B, the simplest ICA model requires at least two mixtures to estimate the two independent sources. However, the first mixture is the watermarked image $\mathbf{y}_{\mathbf{w}}$ derived from (1), but the second mixture is none and unknown in our case. Accordingly, the reference image $\mathbf{y}_{\mathfrak{f}}$ must be generated as the second mixture; it is proposed as a mix of watermarked image $\mathbf{y}_{\mathbf{w}}$ and estimated visible watermark $\hat{\mathbf{w}}$

$$
\mathbf{y}_{\mathrm{f}}(i, j)=k_{3}^{\prime}(i, j) \mathbf{y}_{\mathrm{w}}(i, j)+k_{4}^{\prime}(i, j) \hat{\mathbf{w}}(i, j)
$$

where $k_{3}^{\prime}(i, j)$ and $k_{4}^{\prime}(i, j)$ are the two weighting factors $k_{3}^{\prime}(i, j)+$ $k_{4}^{\prime}(i, j)=1$. Furthermore, $k_{3}^{\prime}(i, j)$ is defined as

$k^{\prime}{ }_{3}(i, j)= \begin{cases}0, & \text { if the }(i, j) \text { th pixel locates at the watermarked area } \\ 1, & \text { elsewhere. }\end{cases}$

This work considers two kinds of visible watermarks: one is the uniform watermark and the other is the linear-gradient watermark. Therefore, the reference image is generated using two different estimation methods. As $\mathbf{y}_{\mathrm{w}}$ is embedded with the uniform watermark, the estimated visible watermark is designed to be a constant image, that is $\hat{\mathbf{w}}(i, j)=1$. For a $K \times L$ linear-gradient watermark, the estimated visible watermark is designed as

$$
\hat{\mathbf{w}}(i, j)=r \times f(i, j, \theta)=r \times\left[\frac{i-1}{K-1} \cos \theta+\frac{j-1}{L-1} \sin \theta\right](6
$$

where $r$ is a factor that adjusts the intensity slope, $\theta$ is the angle of inclination between the direction of the gradient and the horizontal axis, $0 \leq \theta<360,1 \leq i \leq K$, and $1 \leq j \leq L$. The estimated visible watermark is an 8-bit gray-level image and the maximum of $|f(i, j, \theta)|$ in (6) is $\sqrt{2}$, so $r$ satisfies $0<r \leq 255 / \sqrt{2}$.

If the visible watermark is successfully removed, the recovered pixels will be close to the neighboring nonwatermarked pixels. Hence, the error between the pixel values of recovered pixels and those of the neighboring nonwatermarked pixels is measured in order to estimate the proper slope $r$ and the angle $\theta$ for the linear-gradient visible watermark. The difference function is formulated as

$$
\mathrm{DF}=\underset{(i, j) \in c}{\Sigma_{i} \Sigma_{j}}\left|\mathrm{y}_{\mathrm{r}}(i, j)-\mu_{\mathrm{P}}(i, j)\right|^{2}
$$

where $\mathrm{y}_{\mathrm{r}}(i, j)$ is the pixel value of the $(i, j)$ th recovered pixel, and $\mu_{\mathbf{P}}(i, j)$ is an average of all nonwatermarked pixel values in a $5 \times 5$ window $\mathbf{P}$, centered at position $(i, j)$. Let $c$ be the set of coordinate pairs in the interior area $\mathbf{M}_{\text {int }}$

$$
\mathbf{M}_{\text {int }}=\mathbf{M}_{\mathrm{w}}-\left(\mathbf{M}_{\mathrm{w}} \ominus \mathbf{S}_{1}\right)
$$

where $\mathbf{S}_{1}$ is a $3 \times 3$ unitary block and the symbol " $\ominus$ " is the morphology erosion operation. For example, Figs. 2(a) displays a watermarked image and $\mathbf{M}_{\mathrm{w}}$ and $\mathbf{M}_{\text {int }}$ are the watermarked and interior areas, which are shown in Fig. 2(b) and (c), respectively. Fig. 3 presents the difference calculation according to (7). While $\mathbf{y}_{\mathrm{r}}(i, j)$ is close to $\mu_{\mathbf{P}}(i, j)$, the difference DF is low, whereas the boundary between the watermarked and nonwatermarked areas is indistinguishable. In contrast, the high-difference DF indicates that the boundary between the watermarked and nonwatermarked areas is noticeable.

Initially, 432 reference images are generated with 12 slopes and 36 angles $\left(r=\Sigma_{k=1}^{12} 15 \times k\right.$ and $\left.\theta=\Sigma_{k=0}^{35} 10^{\circ} \times k\right)$, and these images are applied to VWR. To calculate the differences of 432 recovered images by (7), the first proper parameters $r_{1}$ and $\theta_{1}$ correspond to the recovered image with minimum difference. Subsequently, 35 reference images with five slopes and seven angles $\left(r=r_{1}+\Sigma_{k=-2}^{2} 3 \times k\right.$ and $\theta=$ $\left.\theta_{1}+\Sigma_{k=-3}^{3} 3^{\circ} \times k\right)$ are generated for VWR. To calculate the differences of 35 recovered images by (7), the second proper parameters $r_{2}$ and $\theta_{2}$ correspond to the recovered image with the minimum difference. Finally, 25 reference images with 5 slopes and 5 angles $\left(r=r_{2}+\right.$ $\Sigma_{k=-2}^{2} k$ and $\left.\theta=\theta_{2}+\Sigma_{k=-2}^{2} 1^{\circ} \times k\right)$ are generated for VWR. To calculate the differences of 25 recovered images by (7), the final proper parameters $r_{\text {final }}$ and $\theta_{\text {final }}$ correspond to the recovered image with the minimum difference as well. Then, not only $r_{\text {final }}$ and $\theta_{\text {final }}$ of the estimated linear-gradient watermark are found, but the image is also 


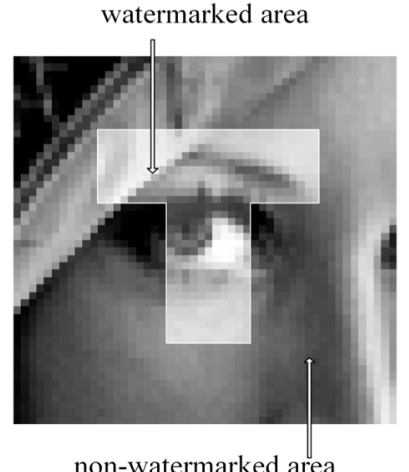

(a)

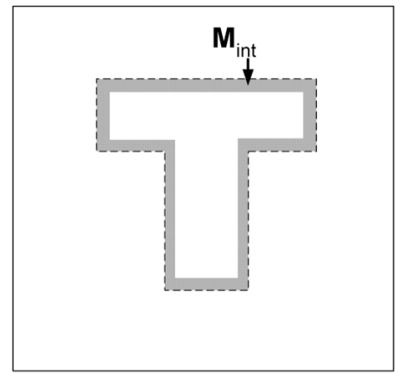

(c)

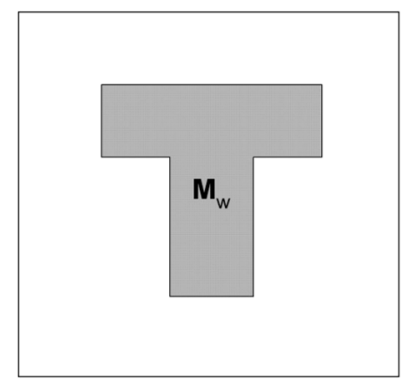

(b)

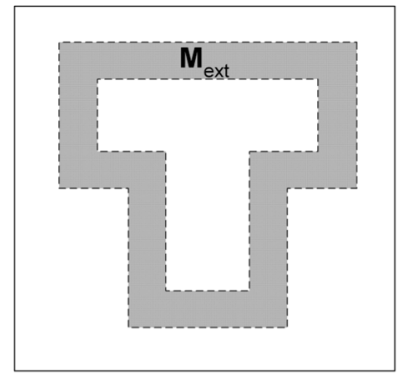

(d)
Fig. 2. (a) Watermarked image. (b) Watermarked area $\mathbf{M}_{\mathbf{w}} \cdot$ (c) Interior area $\mathbf{M}_{\text {int }}$ (d) Exterior area $\mathbf{M}_{\text {ext }}$.

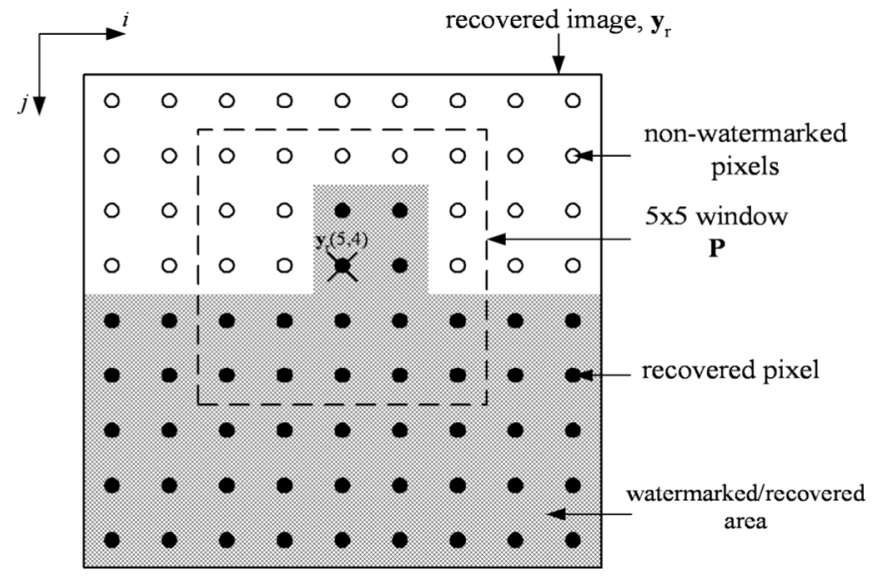

Fig. 3. Diagram presents the difference calculation according to (7). A $5 \times 5$ window $\mathbf{P}(i, j)$ consists of the nonwatermarked and recovered pixels.

recovered. A total of 492 reference images is generated in the above iterative process.

\section{Image Recovery}

Since the watermarked image is a mixture of the host image and the visible watermark, the reference image is generated to be a mix of the watermarked image and estimated visible watermark as in (4). The reference image can also be expressed as another mixture form of the host image and the visible watermark

$$
\begin{aligned}
\mathbf{y}_{\mathrm{f}}(i, j) & =k^{\prime}{ }_{3}(i, j) \mathbf{y}_{\mathbf{w}}(i, j)+k^{\prime}{ }_{4}(i, j) \hat{\mathbf{w}}(i, j) \\
& =k^{\prime}{ }_{3}(i, j)\left[k_{1}(i, j) \mathbf{x}(i, j)+k_{2}(i, j) \mathbf{w}(i, j)\right]+k^{\prime}{ }_{4}(i, j) \hat{\mathbf{w}}(i, j) \\
& =k_{3}(i, j) \mathbf{x}(i, j)+k_{4}(i, j) \mathbf{w}(i, j)
\end{aligned}
$$

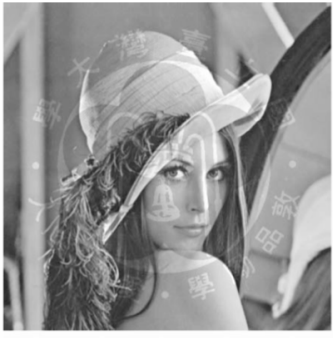

(a)

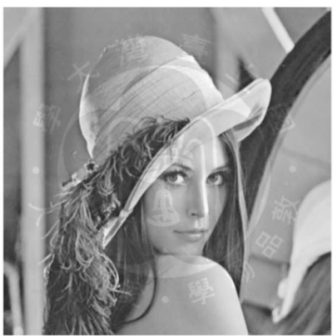

(c)

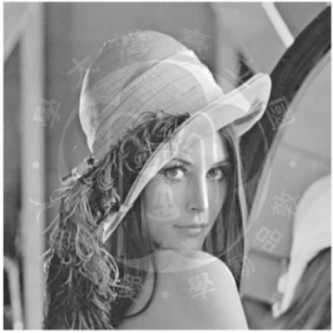

(e)

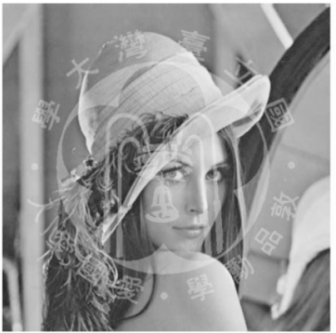

(g)

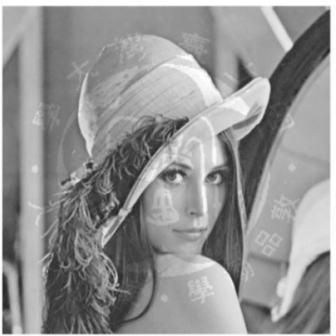

(i)

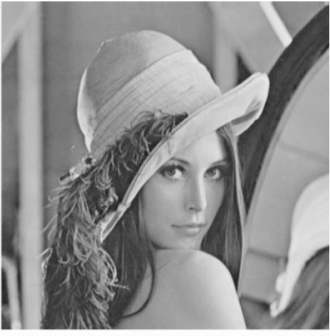

(b)

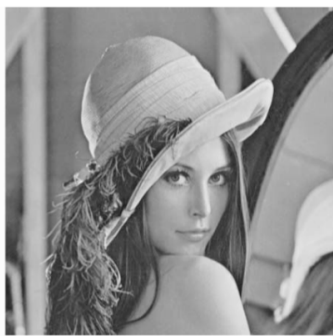

(d)

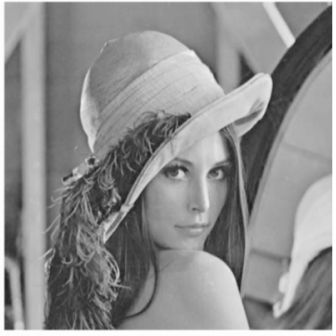

(f)

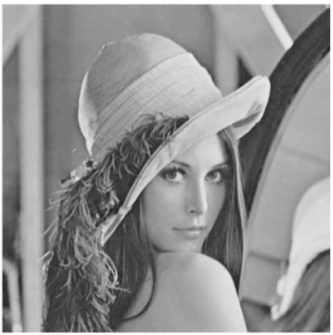

(h)

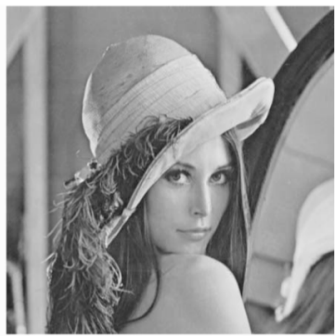

(j)
Fig. 4. Results of uniform watermark removal. The left-hand column presents the watermarked images shown in (a), (c), (e), (g), and (i) by the following five embedding algorithms, which include Braudaway's, Meng's, Mohanty's, Hu's, and Chen's methods. The right-hand column shows that the recovered images by our proposed VWR algorithm correspond to the left-hand column images. The image recovery process takes an average of $1.8 \mathrm{~s}$, and the PSNRs of those recovered images are 48.84, 47.24, 34.38, 43.95, and $37.44 \mathrm{~dB}$ for (b), (d), (f), (h), and (j), respectively.

where $k_{3}^{\prime}(i, j)=k_{3}^{\prime}(i, j) k_{1}(i, j)$ and $k_{4}(i, j)=k_{3}^{\prime}(i, j) k_{2}(i, j)+$ $k_{4}^{\prime}(i, j) \hat{w}(i, j) / \mathbf{w}(i, j)$. When the watermarked image $\mathbf{y}_{\mathrm{w}}$ and the reference image $\mathbf{y}_{\mathrm{f}}$ are obtained, the ICA is utilized to estimate a $2 \times 2$ 


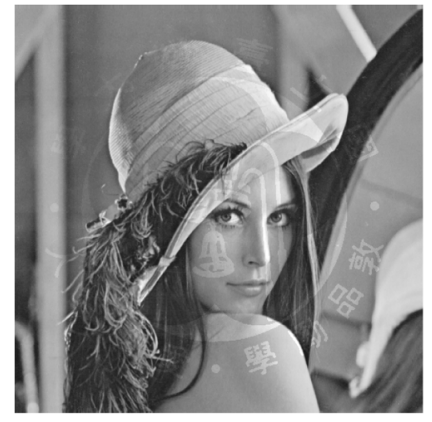

(a)

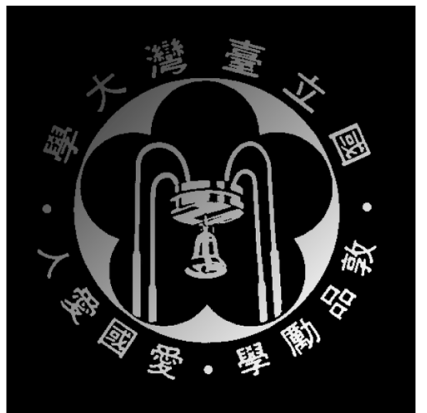

(b)

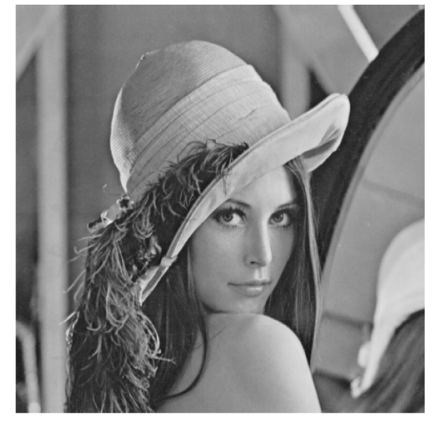

(c)

Fig. 5. Proposed algorithm recovers the linear-gradient watermarked image. (a) Watermarked image with PSNR $=30.97 \mathrm{~dB}$. (b) Linear-gradient watermark with $r=12$ and $\theta=220^{\circ}$. (b) Recovered image with PSNR $=47.22 \mathrm{~dB}$ in $783.30 \mathrm{~s}\left(r_{\text {final }}=8\right.$ and $\left.\theta_{\text {final }}=233^{\circ}\right)$.

TABLE I

MEANS $(\mu)$ AND VARIANCES $\left(\sigma^{2}\right)$ OF THE ORIGINAL, WATERMARKED, AND RECOVERED AREAS ASSOCIATE WITH THE UNIFORM AND LINEAR-GRADIENT WATERMARKS

\begin{tabular}{|c|c|c|c|c|c|c|c|}
\hline \multirow{2}{*}{$\begin{array}{l}\text { Watermark } \\
\text { Types }\end{array}$} & \multirow{2}{*}{$\begin{array}{c}\text { Watermarking } \\
\text { Methods }\end{array}$} & \multicolumn{2}{|c|}{ Original Area } & \multicolumn{2}{|c|}{ Watermarked Area } & \multicolumn{2}{|c|}{ Recovered Area } \\
\hline & & $\mu$ & $\sigma^{2}$ & $\mu$ & $\sigma^{2}$ & $\mu$ & $\sigma^{2}$ \\
\hline \multirow{5}{*}{$\begin{array}{c}\text { Uniform } \\
\text { Watermark }\end{array}$} & Braudaway & \multirow{6}{*}{120.39} & \multirow{6}{*}{2477.7} & 147.48 & 3319.2 & 117.98 & 2450.2 \\
\hline & Meng & & & 139.31 & 2970.9 & 117.96 & 2450.2 \\
\hline & Mohanty & & & 140.09 & 2312.3 & 116.50 & 2508.1 \\
\hline & $\mathrm{Hu}$ & & & 158.49 & 1274.0 & 117.96 & 2449.9 \\
\hline & Chen & & & 146.78 & 3726.4 & 117.95 & 2449.8 \\
\hline $\begin{array}{l}\text { Linear-gradient } \\
\text { Watermark }\end{array}$ & Braudaway & & & 137.11 & 3027.2 & 117.95 & 2448.3 \\
\hline
\end{tabular}

separating matrix $\mathbf{B}$, such that the two source images $\mathbf{X}_{1}$ and $\mathbf{X}_{2}$ can be separated

$$
\left[\begin{array}{l}
\mathbf{X}_{1} \\
\mathbf{X}_{2}
\end{array}\right]=\mathbf{B}\left[\begin{array}{l}
\mathbf{y}_{\mathrm{w}} \\
\mathbf{y}_{\mathrm{f}}
\end{array}\right] \text { and } \mathbf{B}=\left[\begin{array}{ll}
b_{1} & b_{2} \\
b_{3} & b_{4}
\end{array}\right]
$$

The issue in which the two separated source images should be applied to recover the watermarked image is of concern. By the heuristic experiment with 50 images, the solution is determined by two coefficients of $\mathbf{B}, b_{1}$ and $b_{3}$. If $\left|b_{1}\right| \geq\left|b_{3}\right|$, then $\mathbf{X}_{1}$ is the appropriate source image; on the contrary, $\mathbf{X}_{2}$ is the appropriate source image if $\left|b_{3}\right|>\left|b_{1}\right|$. The appropriate source image is called the nonwatermarked source image $\mathbf{X}_{\mathrm{nw}}$.

The mean $\mu_{\mathrm{nw}}$ and variance $\sigma_{\mathrm{nw}}^{2}$ of the watermarked area in $\mathbf{X}_{\mathrm{nw}}$ are calculated as

$$
\mu_{\mathrm{nw}}=\frac{\sum_{i} \sum_{j} \mathbf{X}_{\mathrm{nw}}(i, j) \mathbf{M}_{\mathrm{w}}(i, j)}{\sum_{i} \sum_{j} \mathbf{M}_{\mathrm{w}}(i, j)}
$$

and

$$
\sigma_{\mathrm{nw}}^{2}=\frac{\sum_{i} \sum_{j}\left(\mathrm{X}_{\mathrm{nw}}(i, j)-\mu_{\mathrm{nw}}\right)^{2} \mathrm{M}_{\mathrm{w}}(i, j)}{\sum_{i} \sum_{j} \mathrm{M}_{\mathrm{w}}(i, j)} .
$$

TABLE II

SEPARATING MATRICES AND PSNR OF THE RECOVERED IMAGES,

\begin{tabular}{|c|c|c|c|c|c|}
\hline & Braudaway & Meng & Mohanty & $\mathrm{Hu}$ & Chen \\
\hline JADE & {$\left[\begin{array}{rr}0.5791 & 0.5796 \\
0.9899 & -0.9896\end{array}\right]$} & {$\left[\begin{array}{rr}0.5972 & 0.5410 \\
1.0290 & -1.0604\end{array}\right]$} & {$\left[\begin{array}{rr}0.5857 & 0.5578 \\
1.0228 & -1.0383\end{array}\right]$} & {$\left[\begin{array}{rr}0.6541 & 0.5156 \\
0.9123 & -0.9972\end{array}\right]$} & {$\left[\begin{array}{cc}0.5674 & 0.5920 \\
0.9949 & -0.9804\end{array}\right]$} \\
\hline FastICA & {$\left[\begin{array}{rr}-0.5940 & -0.5646 \\
-0.9810 & 0.9982\end{array}\right]$} & {$\left[\begin{array}{r}1.0165-1.0719 \\
-0.6195-0.5178\end{array}\right]$} & {$\left[\begin{array}{lr}-0.6041 & -0.5389 \\
-1.0120 & 1.0482\end{array}\right]$} & {$\left[\begin{array}{ll}-0.9265 & 0.9855 \\
-0.6338 & -0.5374\end{array}\right]$} & {$\left[\begin{array}{r}-0.5877-0.5718 \\
0.9830-0.9923\end{array}\right]$} \\
\hline SOBI & {$\left[\begin{array}{ll}-0.8328 & -0.2798 \\
-0.7885 & 1.1122\end{array}\right]$} & {$\left[\begin{array}{ll}-0.8700 & -0.2106 \\
-0.8126 & 1.1716\end{array}\right]$} & {$\left[\begin{array}{rr}-0.8571 & -0.2328 \\
-0.8090 & 1.1554\end{array}\right]$} & {$\left[\begin{array}{rr}-0.8178 & -0.3134 \\
-0.7689 & 1.0779\end{array}\right]$} & {$\left[\begin{array}{rr}-0.8308 & 0.2833 \\
-0.7883 & 1.1097\end{array}\right]$} \\
\hline PSNR (dB) & 48.84 & 47.24 & 34.38 & 43.95 & 44.91 \\
\hline
\end{tabular}
VWR IS INTEGRATED WITH THREE ICA APPROACHES TO RECOVER FIVE VISIBLE WATERMARKED IMAGES

$\mathbf{M}_{\text {ext }}$ is the exterior area, which surrounds the watermarked area shown in Fig. 2(d)

$$
\mathbf{M}_{\mathrm{ext}}=\left(\mathrm{M}_{\mathrm{w}} \oplus \mathbf{S}_{2}\right)-\mathbf{M}_{\mathrm{w}}
$$

where $\mathbf{S}_{2}$ is an $11 \times 11$ unitary block and the symbol " $\oplus$ " is the morphology dilation operation. Let $\mu_{\text {ext }}$ and $\sigma_{\text {ext }}^{2}$ be the mean and variance of the exterior area in the watermarked image $\mathbf{y}_{\mathrm{w}}$

$$
\mu_{\mathrm{ext}}=\frac{\sum_{i} \sum_{j} \mathbf{y}_{\mathrm{w}}(i, j) \mathbf{M}_{\mathrm{ext}}(i, j)}{\sum_{i} \sum_{j} \mathbf{M}_{\mathrm{ext}}(i, j)}
$$

and

$$
\sigma_{\mathrm{ext}}^{2}=\frac{\sum_{i} \sum_{j}\left(\mathbf{y}_{\mathrm{w}}(i, j)-\mu_{\mathrm{out}}\right)^{2} M_{\mathrm{ext}}(i, j)}{\sum_{i} \sum_{j} \mathbf{M}_{\mathrm{ext}}(i, j)} .
$$

Although the exterior area surrounds the watermarked area, both areas should have similar means and variances before the visible watermark is added to the image. Consequently, the mean and standard deviation of the watermarked area in $\mathbf{X}_{\mathrm{nw}}$ are adjusted, and the recovered image is defined in (14), shown at the bottom of the page. If the quality of the recovered image is unsatisfactory, then the recovered image replaces the watermarked image and the above VWR algorithm is iteratively processed again.

$$
\mathbf{y}_{\mathrm{r}}(i, j)= \begin{cases}\frac{\sigma_{\mathrm{ext}}}{\sigma_{\mathrm{nw}}}\left(\mathbf{X}_{\mathrm{nw}}(i, j)-\mu_{\mathrm{nw}}\right)+\mu_{\mathrm{ext}}, & \text { if pixel locates at the watermarked area } \\ \mathbf{y}_{\mathrm{w}}(i, j), & \text { elsewhere }\end{cases}
$$




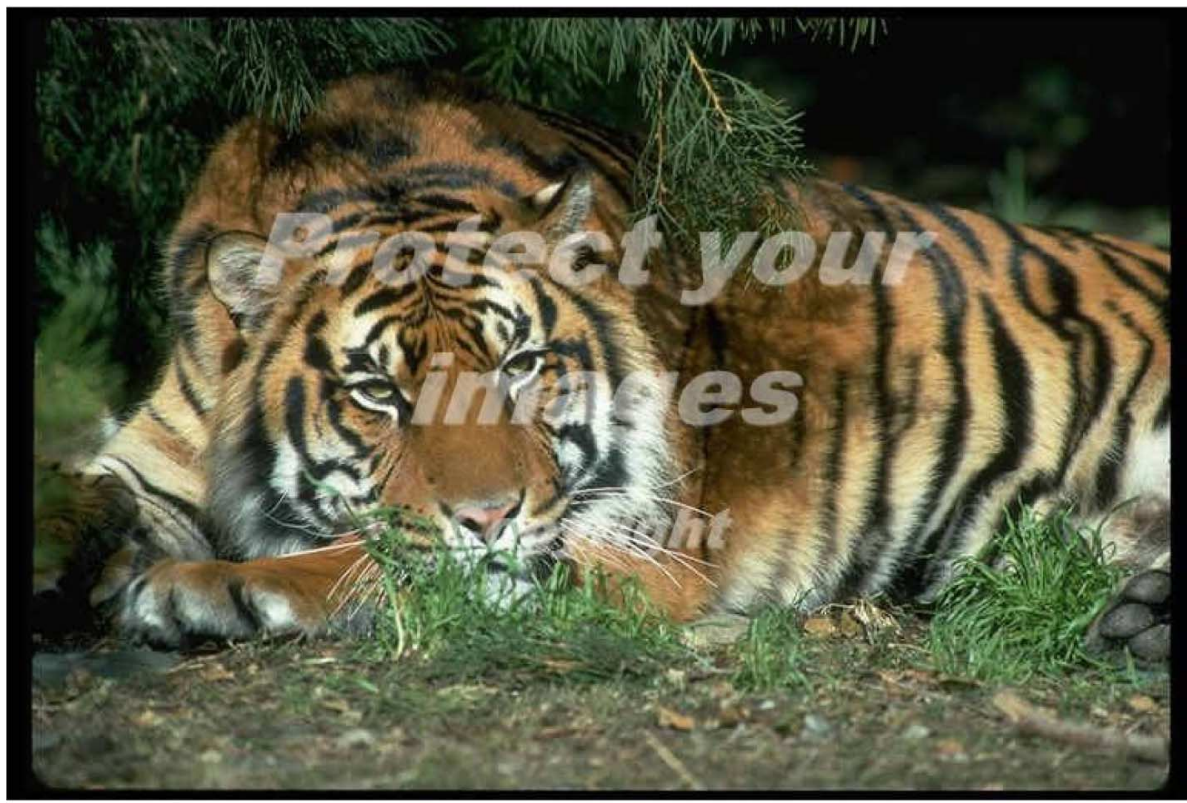

(a)

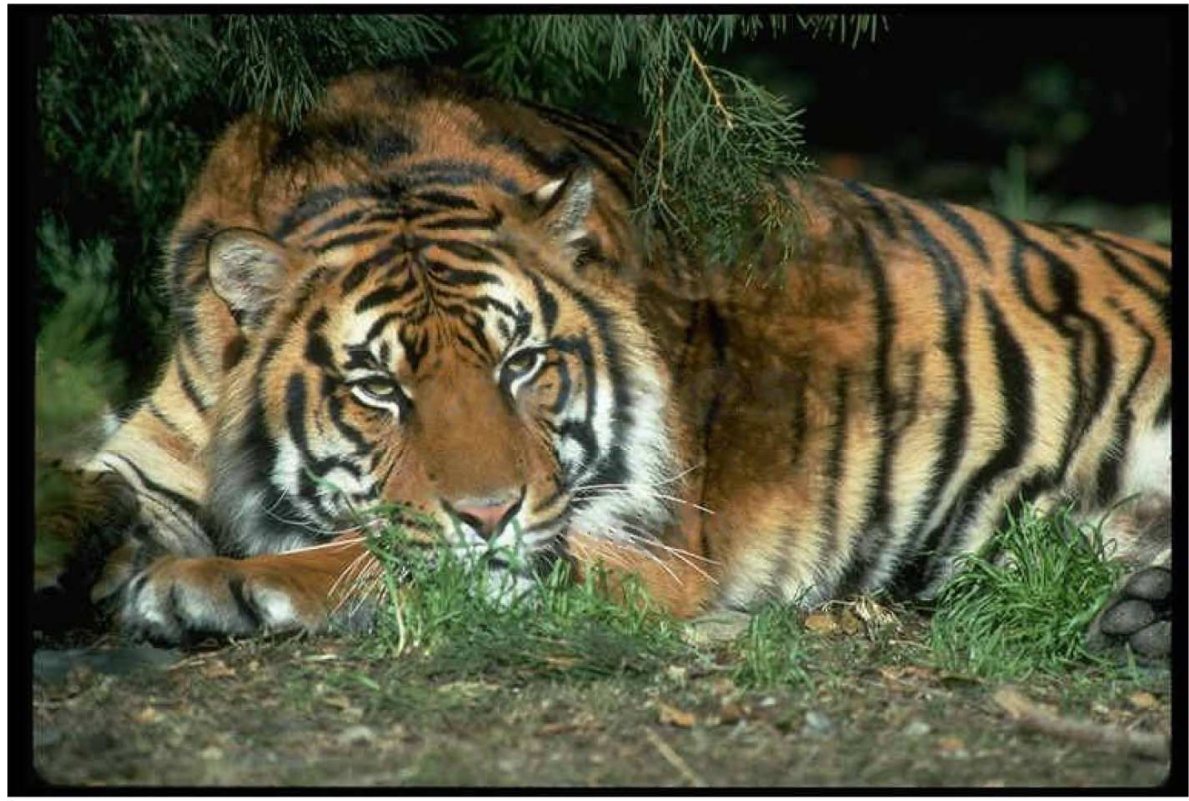

(b)

Fig. 6. Recovering the public domain image. (a) $768 \times 512$ watermarked image is embedded with a small watermark [17]. (b) Recovered image with a process time of $4.77 \mathrm{~s}$.

\section{EXPERIMENTAL RESULTS}

The experiments focus on three issues to show that VWR is an effective image recovery algorithm for removing the visible watermark. These three issues include different visible watermarking methods, various ICA approaches, and the blind removal of visible watermarks. The threshold parameters for segmenting the watermarked area are $\tau=8$ and $\Delta \tau=4$

\section{A. Visible Watermarking Methods}

The $512 \times 512$ gray-level image is tested in the experiment. First, five test images are embedded with five $512 \times 512$ uniform visible watermarks using the different watermarking methods of Braudaway et al. [5], Meng [6], Mohanty et al. [8], Hu [9], and Chen [10]. JADE [11] of the ICA approach is used in our proposed VWR algorithm. The left-hand column of Fig. 4 shows these five watermarked images, and the right-hand column of Fig. 4 shows the recovered images. The image recovery process takes an average of $1.8 \mathrm{~s}$, and the peak signal-to-noise ratios (PSNRs) of those recovered images are 48.84, 47.24, 34.38, 43.95, and $37.44 \mathrm{~dB}$ for Fig. 4(b), (d), (f), (h), and (j), respectively. In the second experiment, a linear-gradient watermark [shown in Fig. 5(b)] with the slope $r=12$ and angle $\theta=220^{\circ}$ is embedded in the test image by Braudaway's method. The PSNRs of the watermarked and recovered images are 30.97 and $47.22 \mathrm{~dB}$, and are displayed in Fig. 5(a) and (c), respectively. The process takes 783.30 $\mathrm{s}$ and the parameters are estimated as $r_{\text {final }}=8$ and $\theta_{\text {final }}=233^{\circ}$. Table I lists the means and variances of the original, watermarked, and recovered areas associated with the uniform and linear-gradient watermarks. The mean and the variance of the recovered area are close to those of the original area, and it indicates that the recovered area is similar to the original area. 


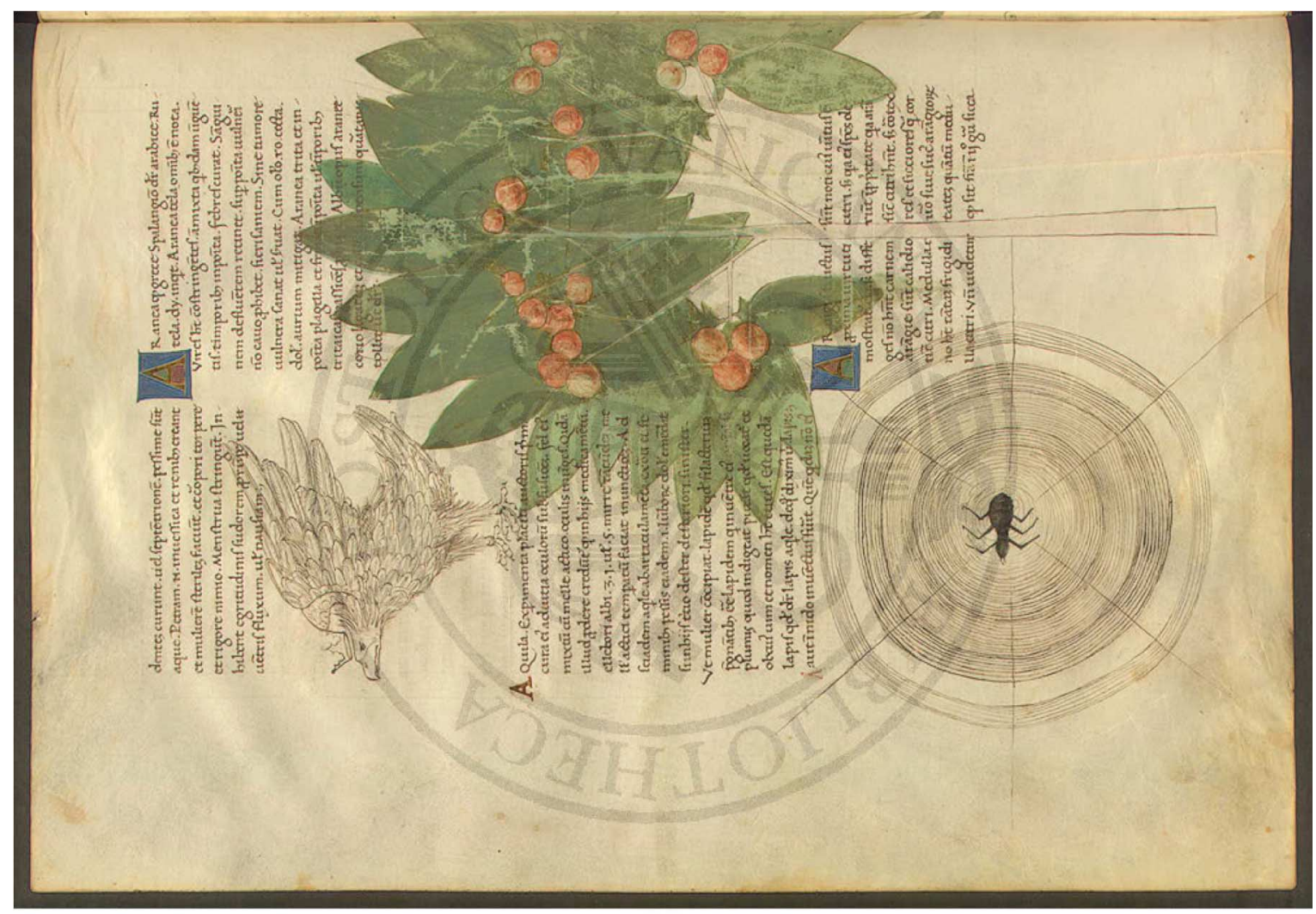

(a)

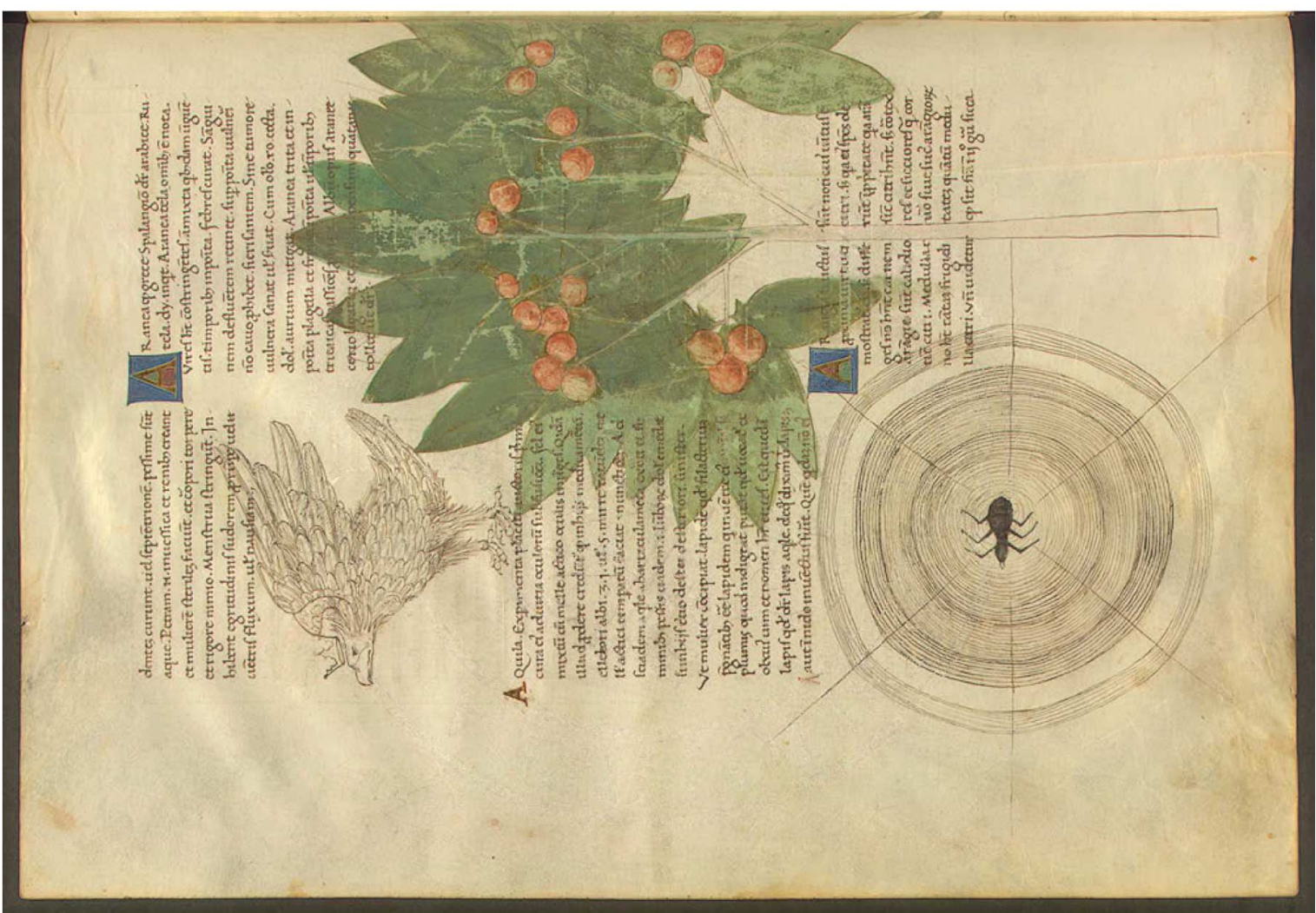

(b)

Fig. 7. Recovering the public domain image. (a) $1000 \times 688$ watermarked image is embedded with a large watermark [18]. (b) Recovered image with a process time of $10.75 \mathrm{~s}$. 


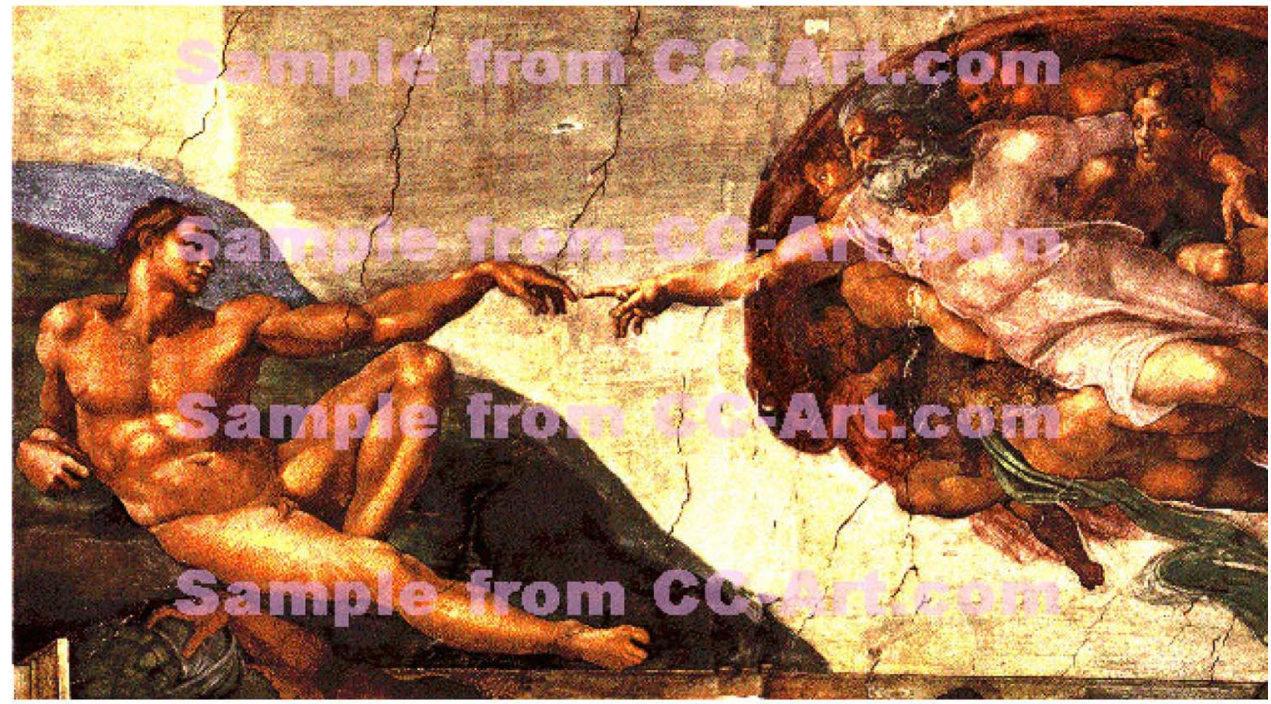

(a)

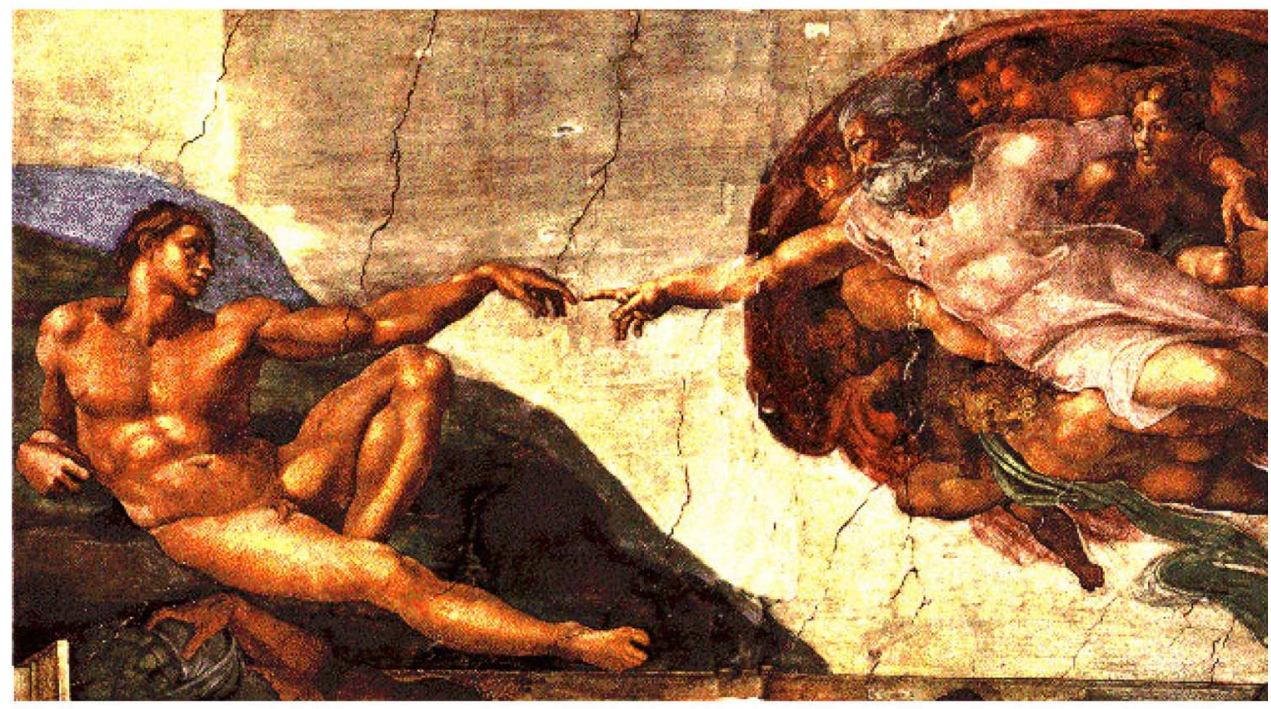

(b)

Fig. 8. Recovering the public domain image. (a) $640 \times 353$ watermarked image is embedded with a color watermark [19]. (b) Recovered image with process time in $4.92 \mathrm{~s}$.

\section{B. ICA Approaches}

Three ICA approaches, which include JADE, FastICA, and SOBI, are examined in our experiments. The MATLAB source codes of these approaches are downloaded from [14]-[16]. VWR is integrated with each ICA approach to recover five watermarked images [as shown in Fig. 4(a), (c), (e), (g), and (i)], and 15 recovered images are obtained. Table II lists the separating matrices and PSNR values of these 15 recovered images. The high PSNR value demonstrates that VWR is independent of the adopted ICA approaches, and successfully removes the visible watermarks embedded with the currently existing visible watermarking methods.

\section{Blind Removal of Visible Watermarks}

Numerous public domain images are available at various websites, and some of them are visible watermarks embedded with unknown watermarking methods [17]-[21]. In the final experiment, the embedded watermarks are assumed to be uniform, and these visible watermarks are removed successfully and blindly from the public images without knowing the watermarking methods in advance. Fig. 6(a) presents an image embedded with the small watermark [17], the $768 \times 512$ recovered image with a process time of $4.77 \mathrm{~s}$ is shown in Fig. 6(b). Fig. 7(a) displays an image embedded with the large watermark [18], the $1000 \times 688$ recovered image with process time $10.75 \mathrm{~s}$ is shown in Fig. 7(b). The final image is shown in Fig. 8(a), which is embedded with the color watermark [19], the $640 \times 353$ recovered image with process time $4.92 \mathrm{~s}$ is shown in Fig. 8(b).

\section{CONCLUSION}

This paper presents a novel image recovery algorithm for removing the visible watermarks. The proposed algorithm not only removes the specified visible uniform and linear-gradient watermarks, but also successfully and blindly recovers the public domain images. Furthermore, three independent component analysis approaches and five visible watermarking methods are examined in the visible watermark removal algorithm, and the experimental results demonstrate that the proposed algorithm is independent of both the adopted ICA approach and the visible watermarking method. All programs are executed in MATLAB software using a $1.5-\mathrm{GHz}$ Pentium-M processor. 


\section{REFERENCES}

[1] M. Bertalmio, G. Sapiro, V. Caselles, and C. Ballester, "Image inpainting," SIGGRAPH, pp. 417-424, Aug. 2000.

[2] J. Sun, L. Yuan, J. Jia, and H.-Y. Shum, "Image completion with structure propagation," SIGGRAPH, pp. 861-868, Aug. 2005.

[3] S.-C. Pei, Y.-C. Zeng, and C.-H. Chang, "Virtual restoration of ancient Chinese paintings using color contrast enhancement and lacuna texture synthesis," IEEE Trans. Image Process., vol. 13, no. 3, pp. 416-429, Mar. 2004.

[4] C.-H. Huang and J.-L. Wu, "Attacking visible watermarking schemes," IEEE Trans. Multimedia, vol. 6, no. 1, pp. 16-30, Feb. 2004.

[5] G. Braudaway, K. A. Magerlein, and F. Mintzer, "Protecting publicly available images with a visible image watermark," in Proc. SPIE, Int. Conf. Electronic Imaging, Feb. 1-2, 1996, vol. 2659, pp. 126-133.

[6] J. Meng and S.-F. Chang, "Embedding visible video watermarks in the compressed domain," in Proc. ICIP, Oct. 4-7, 1998, vol. 1, pp. 474-477.

[7] S. P. Mohanty, K. R. Ramakrishnan, and M. S. Kankanhalli, "A dual watermarking technique for image," in Proc. 7th ACM Int. Multimedia Conf., Oct./Nov. 1999, vol. 2, pp. 49-51.

[8] S. P. Mohanty, K. R. Ramakrishnan, and M. S. Kankanhalli, "A DCT domain visible watermarking technique for image," in Proc. IEEE Int. Conf. Multimedia and Expo., Jul./Aug. 2000, vol. 2, pp. 1029-1032.

[9] Y. Hu and S. Kwong, "Wavelet domain adaptive visible watermarking," Electron. Lett., vol. 37, no. 20, pp. 1219-1220, Sep. 2001.
[10] P.-M. Chen, "A visible watermarking mechanism using a statistic approach," in Proc. 5th Int. Conf. Signal Processing, 2000, vol. 2, pp. 910-913.

[11] J. F. Cardoso and A. Souloumiac, "Blind beamforming for non Gaussian signals," Proc. Inst. Elect. Eng. F, vol. 140, no. 6, pp. 362-370, Dec. 1993.

[12] A. Hyvarinen and E. Oja, "Independent component analysis: Algorithms and applications," Neural Netw., vol. 13, pp. 411-430, May/Jun. 2000.

[13] A. Belouchrani, K. Abed-Meraim, J. F. Cardoso, and E. Moulines, "A blind source separation technique using second order statistics," IEEE Trans. Signal Process., vol. 45, no. 2, pp. 434-444, Feb. 1997.

[14] JADE Program. [Online]. Available: http://sig.enst.fr/ cardoso/stuff. $\mathrm{html}$.

[15] FastICA Program. [Online]. Available: http://www.cis.hut.fi/projects/ ica/fastica/.

[16] SOBI Program. [Online]. Available: http://www.tsi.enst.fr/icacentral/ icalistArchive/2004/0727.html.

[17] [Online]. Available: http://www.fvs.co.uk/fvshome/watermhelp.asp.

[18] [Online]. Available: http://www.dlib.org/dlib/december97/ibm/12lotspiech.html.

[19] [Online]. Available: http://www.biblepicturegallery.com/.

[20] [Online]. Available: http://www.blinkred.com/gallery/static/home. asp.

[21] [Online]. Available: http://earthwindow.com/mola.html. 